\title{
General-relativistic rotation: Self-gravitating fluid tori in motion around black holes
}

\author{
Janusz Karkowski, Wojciech Kulczycki, Patryk Mach, Edward Malec, Andrzej Odrzywołek, and Michał Piróg \\ Instytut Fizyki im. Mariana Smoluchowskiego, Uniwersytet Jagielloński, Eojasiewicza 11, 30-348 Kraków, Poland
}

\begin{abstract}
We obtain from the first principles a general-relativistic Keplerian rotation law for self-gravitating disks around spinning black holes. This is an extension of a former rotation law that was designed mainly for toroids around spinless black holes. We integrate numerically axial stationary Einstein equations with self-gravitating disks around spinless or spinning black holes; that includes the first ever integration of the Keplerian selfgravitating tori. This construction can be used for the description of tight black hole-torus systems produced during coalescences of two neutron stars or modelling of compact active galactic nuclei.
\end{abstract}

PACS numbers: 04.20.-q, 04.25.Nx, 04.40.Nr, 95.30.Sf

\section{INTRODUCTION}

Keplerian rotation is common in rotating astrophysical systems. Quasistationary fluid tori rotating around black holes may emerge in binary neutron stars or black holeneutron star mergers ([1] and references therein). There is a recent detection of gravitational waves coming from such a binary merger [2]. Tori in these systems seem to be relatively massive and yet rotate with the Keplerian velocity [3]. We proposed in [7] a general-relativistic (GR in what follows) differential rotation law capable of describing a stationary system consisting of a selfgravitating disk circulating around a spinless or spinning black hole (BH hereafter). Its interesting feature (absent in other proposals [8-13]) is that the nonrelativistic limit exactly yields the Newtonian angular velocity $\Omega=w / d^{\lambda}$, where $w$ is a constant. Here $0 \leq \lambda \leq 2$, and $d$ is the Euclidean distance from the rotation axis. This limit includes the Keplerian rotation law with $\lambda=3 / 2$. This rotation law has been recently investigated within the first post-Newtonian (1PN) approximation, for a spinless $\mathrm{BH}$ [14.

The main goals of this paper are: (i) to show a new rotation law that can be more adequate for Keplerian systems consisting of a spinning $\mathrm{BH}$ and a disk; (ii) describe numerically a polytropic disk, using both versions of rotation laws - that of [7] and the new one -in the full Einstein theory within the puncture framework as formulated by Shibata 15.

We shall give a concise report on the formalism and obtained results; details are given in a longer accompanying paper [16]. In numerical calculations we investigated several values of the spin parameter in the interval $a \in[-0.9,0.9]$ and mainly light disks, that include cases shown to be of interest in recent simulations of coalescences of neutron stars [1, 17].

\section{EQUATIONS}

We choose a stationary metric

$$
d s^{2}=-\alpha^{2} d t^{2}+r^{2} \sin ^{2} \theta \psi^{4}(d \phi-\beta d t)^{2}
$$

$$
+\psi^{4} e^{2 q}\left(d r^{2}+r^{2} d \theta^{2}\right)
$$

We put $G=c=1$. Here $t$ is the time coordinate, and $r, \theta, \phi$ are spherical coordinates. We assume axial symmetry and employ the stress-momentum tensor $T^{\alpha \beta}=\rho h u^{\alpha} u^{\beta}+p g^{\alpha \beta}$, where $\rho$ is the baryonic restmass density, $h$ is the specific enthalpy, and $p$ is the pressure. We assume that $p(\rho)=K \rho^{\frac{4}{3}}$, but our analysis can be done for any barotropic equation of state. The 4-velocity $\left(u^{\alpha}\right)=\left(u^{t}, 0,0, u^{\phi}\right)$ is normalized, $u_{\beta} u^{\beta}=-1$. The angular velocity $\Omega=u^{\phi} / u^{t}$. We use Einsteinhydrodynamic equations as formulated by Shibata (see Sec. II in [15] or Secs. II and III in [16]). It is well known that GR Euler equations are solvable under an integrability condition 8, 9. In accordance with that, we assume that the angular momentum per unit inertial mass $\rho h$ [18, $j \equiv u_{\phi} u^{t}$ depends only on the angular velocity $\Omega$, $j \equiv j(\Omega)$.

The metric functions satisfy equations (44)-(48) in [15], with Shibata's boundary conditions on the horizon of the central BH, at the symmetry axis and at spatial infinity. We used the Kerr metric - the only available analytic solution - for testing the correctness of numerical codes. That led to a somewhat different numerical implementation of some of the boundary conditions of [15], in order to get the Kerr solution with the best accuracy in the absence of the torus (see [16] for details).

The $\mathrm{BH}$ is surrounded by a minimal two-surface $S_{\mathrm{BH}}$ (on a fixed hypersurface of constant time). Its area $A_{\mathrm{H}}$ defines the irreducible mass $M_{\mathrm{irr}}=\sqrt{\frac{A_{\mathrm{H}}}{16 \pi}}$ and its angular momentum $J_{\mathrm{H}}$ follows from the Komar expression $J_{\mathrm{H}}=$ $\frac{1}{4} \int_{0}^{\pi / 2} \frac{r^{4} \psi^{6}}{\alpha} \partial_{r} \beta \sin ^{3} \theta d \theta$. The angular momentum is prescribed rigidly on the event horizon $S_{\mathrm{BH}}$-it is given by data taken from the Kerr solution (with two parameters: mass $m$ and the spin parameter $\left.a=J_{\mathrm{H}} / m\right)$, and it is independent of the content of mass in the torus. The mass of the $\mathrm{BH}$ is then defined as $M_{\mathrm{BH}}=M_{\mathrm{irr}} \sqrt{1+\frac{J_{\mathrm{H}}^{2}}{4 M_{\mathrm{irr}}^{4}}}$. Another possible choice of the $\mathrm{BH}$ mass is in terms of the circumferential radius $r_{\mathrm{C}}$ of $S_{\mathrm{BH}}$ at the symmetry plane $\theta=\pi / 2: \quad M_{\mathrm{C}}=r_{\mathrm{C}} / 2$. We observed that in numerical calculations $M_{\mathrm{C}}$ and $M_{\mathrm{BH}}$ differ by significantly less than $1 \%$ [16] this is consistent with findings of Shibata [15]. 
The asymptotic mass $M_{\mathrm{ADM}}$ is defined as in [15. The mass of tori is defined by $M_{\mathrm{T}} \equiv M_{\mathrm{ADM}}-M_{\mathrm{BH}}$.

In 7] we have got the rotation law for the motion around central bodies

$$
j(\Omega) \equiv \frac{\tilde{w}^{1-\delta} \Omega^{\delta}}{1-\kappa \tilde{w}^{1-\delta} \Omega^{1+\delta}+\Psi} ;
$$

here $\Psi$ is of the order of the binding energy per unit baryonic mass, and $\kappa=\frac{1-3 \delta}{1+\delta}$. This formula followed from an "educated guesswork", with three basic elements: (i) the rotation law should have the right (monomial) form in the Newtonian limit; (ii) the $1 \mathrm{PN}$ correction to the Bernoulli equation should have the right form; (iii) the first post-Newtonian (1PN) correction to the angular velocity should be uniquely defined. The $1 \mathrm{PN}$ analysis of the Bernoulli equation yields that (2) is admissible for any $\kappa$. The fact that $\kappa$ can be arbitrary, is unfortunate, because its values influence the angular velocity $\Omega$ - a quantity that can be observable. There exists, however, a physical constraint, that follows from the fact that a massless disk of dust is just a collection of test particles in a circular motion. Therefore such a disk in the Schwarzschild geometry must exactly satisfy the Einstein-Bernoulli equations, with the angular velocity $\Omega=\sqrt{M_{\mathrm{BH}}} / r_{\mathrm{C}}^{3 / 2}$. That implies $\kappa=\frac{1-3 \delta}{1+\delta}$, and the relation between $\kappa$ and $\delta$ becomes unique. The full reasoning is described in [7. Let us remark that spins of BH's do not appear in the $1 \mathrm{PN}$ approximation, and therefore (2) might be expected to apply only to systems with spin-less BH's. Numerical analysis suggests that it is valid also for spinning BH's (see below). Simple rescaling $w^{1-\delta}=\frac{\tilde{w}^{1-\delta}}{1+\Psi}$ transforms (2) into

$$
j(\Omega) \equiv \frac{w^{1-\delta} \Omega^{\delta}}{1-\kappa w^{1-\delta} \Omega^{1+\delta}},
$$

where $w, \delta$ and $\kappa=\frac{1-3 \delta}{1+\delta}$ are parameters [19]. Note that only the value of $\delta \in(-\infty, 0)$ is arbitrary, and that $w$ is obtained as a part of the solution. The cases $\delta=-1, \delta=-1 / 3$ and $\delta=0$ correspond-in the Newtonian limit - to the constant linear velocity, the Keplerian rotation, and the constant angular momentum density, respectively. Let us stress, that in this construction the central body can be a spinless $\mathrm{BH}$, but equally well there can be no central body. We believe also, that this differential rotation can be adapted to rotating stars. The rotation law (3) can be understood as the generalrelativistic version of the Poincaré-Wavre condition for fluids undergoing monomial rotation, $\Omega=w / d^{\lambda}$, in Newtonian gravity.

We performed dozens of numerical runs with different pairs $\left(\delta, \kappa=\frac{1-3 \delta}{1+\delta}\right)$ (where $\delta \in[-0.99,0]$ ) and, remarkably, there always existed solutions. This is reported below.

The rotation curves $\Omega(r, \cos \theta)$ ought to be recovered from $j(\Omega)=u_{\phi} u^{t}$ :

$$
\frac{w^{1-\delta} \Omega^{\delta}}{1-\kappa w^{1-\delta} \Omega^{1+\delta}}=\frac{V^{2}}{(\Omega+\beta)\left(1-V^{2}\right)},
$$

where the square of the linear velocity reads $V^{2}=$ $r^{2} \sin ^{2} \theta(\Omega+\beta)^{2} \frac{\psi^{4}}{\alpha^{2}}$. The Euler equations reduce to a GR integro-algebraic Bernoulli equation, that embodies the hydrodynamic information carried by the continuity equations $\nabla_{\mu} T^{\mu \nu}=0$ and the baryonic mass conservation $\nabla_{\mu}\left(\rho u^{\mu}\right)=0$. It becomes [7]

$$
h \alpha \sqrt{1-V^{2}}\left(1-\kappa w^{1-\delta} \Omega^{1+\delta}\right)^{-\frac{1}{(1+\delta) \kappa}}=C .
$$

\section{TORI IN THE KEPLERIAN ROTATION AROUND SPINNING BLACK HOLES}

Below we obtain analytically a generalization of the rotation curve (3) that describes tori in motion around spinning BH's. It is known that test particles can rotate along circular orbits $r=$ const in the Kerr geometry. That means that there exists a testlike disk made of dust, that moves circularly and lies on the plane $\theta=\pi / 2$. The radial variable $r$ of each disk particle can be expressed in terms of its angular velocity $\Omega(r)=\frac{8 r^{3 / 2}}{\left((2 r+1)^{2}-a^{2}\right)^{3 / 2}+8 a r^{3 / 2}}$. Metric functions $\psi, \beta, \alpha, q$ of the Kerr geometry depend only on the variable $r$, on the disk's surface, hence they can be expressed entirely in terms of $\Omega$. Thus the angular momentum density

$$
j_{\mathrm{K}}=u_{\phi} u^{t}=\frac{r^{2}(\Omega+\beta) \frac{\psi^{4}}{\alpha^{2}}}{1-(\Omega+\beta)^{2} \frac{r^{2} \psi^{4}}{\alpha^{2}}}
$$

becomes a function $j_{\mathrm{K}}(\Omega)$ of the angular velocity $\Omega$. Let us now define a function $F(\Omega)$ as a solution of

$$
\frac{d}{d \Omega} \ln (1-F(\Omega))=-2 j_{\mathrm{K}}(\Omega)
$$

from that we get after lengthy calculations 16 .

$$
F(\Omega)=a^{2} \Omega^{2}+3 m^{\frac{2}{3}} \Omega^{\frac{2}{3}}(1-a \Omega)^{\frac{4}{3}} .
$$

Here $m$ is the mass of the Kerr BH and the formula describes circular motions of massless disks of dust. The parameter $a$ in $(8)$ is just the spin parameter of the Kerr $\mathrm{BH}$.

After finding (7), we discovered a much simpler derivation in Boyer-Lindquist coordinates (the radial coordinate $r_{\mathrm{BL}}$ is different from that used before). The angular velocity reads $\Omega=\frac{\sqrt{m}}{ \pm r_{\mathrm{BL}}^{3 / 2}+a \sqrt{m}}$ (formula (2.16) in [20]); that gives $r_{\mathrm{BL}}=\frac{m^{1 / 3}(1-a \Omega)^{2 / 3}}{\Omega^{2 / 3}}$. Replacing $r_{\mathrm{BL}}$ in $j=u_{\phi} u^{t}=-\frac{g_{0 \phi}+g_{\phi \phi} \Omega}{g_{00}+2 g_{0 \phi} \Omega+g_{\phi \phi} \Omega^{2}}$, one arrives at 7 and 8 .

The application of this approach to the circular motion of the massless disk of dust in Schwarzschild geometry yields $r_{\mathrm{C}}=w^{2 / 3} \Omega^{-2 / 3}$ and $F=3 w^{4 / 3} \Omega^{2 / 3}$; this agrees with (3) for the Keplerian motion.

The rotation curves $\Omega(r, \cos \theta)$ ought to be found from

$$
-\frac{3}{2 \kappa} \frac{d}{d \Omega} \ln \left(1-\frac{\kappa}{3} F(\Omega)\right)=\frac{V^{2}}{(\Omega+\beta)\left(1-V^{2}\right)} .
$$


It is clear from the construction that test disks of dust in the Kerr geometry (with $\kappa=3$ ) satisfy Eq. (9), Einstein equations and the Bernoulli equation

$$
h \alpha \sqrt{1-V^{2}}\left(1-\frac{\kappa}{3} F(\Omega)\right)^{-1 / 2}=C .
$$

We conjecture that

$$
j(\Omega) \equiv-\frac{3}{2 \kappa} \frac{d}{d \Omega} \ln \left(1-\frac{\kappa}{3}\left(a^{2} \Omega^{2}+3 w^{\frac{4}{3}} \Omega^{\frac{2}{3}}(1-a \Omega)^{\frac{4}{3}}\right)\right)
$$

is the rotation law for a heavy torus rotating around a black hole with the spin parameter equal to the parameter $a$ that appears in (11).

$\kappa$ might be regarded as a free parameter in the rotation law (11), but its set of allowed values centers closely around 3. Moreover, the choice $\kappa=3$ always yielded solutions in our numerical calculations. The parameter $w$ in turn is the only unknown and it can be obtained in the process of solving the problem.

Our numerical method follows closely the scheme proposed in [15], with only few modifications [16. The most important one is due to a different rotation law - the replacement of one rotation curve by another in the numerical programme can be quite difficult and might require considerable work. Equation (11) of [15] is replaced with our Eq. (5), which is equivalent to Eq. (A11) of [15, and which suits better the convention with $j=u_{\phi} u^{t}$. Our implementation is described, with a detailed account of the boundary conditions, in [16].

The solution is specified by setting 7 main parameters: the mass $m$ ( $m=1$ in forthcoming examples) and spin $a$ defined as in 15 - they describe the BH; the inner and outer coordinate radii of the disk at the equatorial plane: $r_{1}$ and $r_{2}$, respectively; the maximum of the rest-mass density within the disk $\rho_{0}$; parameters $\delta$ and $\kappa$ in (3). We check the virial condition of [15 - that the Komar and asymptotic masses are equal. We performed several tests of numerical codes 16.

\section{NUMERICAL RESULTS: TORI AROUND SPINLESS BLACK HOLES, ROTATION CURVE} (3)

We shall describe 6 solutions, with the following pairs of parameters, $\left(\delta, \kappa=\frac{1-3 \delta}{1+\delta}\right)$ : $[(-0.99,397) ;(-0.8,17) ;(-0.6,7) ;(-0.4,11 / 3) ;(-0.2,2)$; $(0,1)]$. It is remarkable, that they exist exactly at sets of numbers $(\delta, \kappa(\delta))$ predicted by [7]-Eq. (3) does not have any free parameters in the case of spinless BH's. Profiles of the obtained tori are shown in Fig. 1. They flatten with the decrease of the parameter $\delta$, maximal values of their mass density increase, by a factor of 2 , (from 0.000454 at $\delta=0$ to 0.000879 at $\delta=-0.99$ ) while their maximal specific enthalpy decreases rather moderately (from 1.0348 at $\delta=0$ to 1.0273 at $\delta=-0.99$ ).
Table $[$ shows a sample of results concerning the Keplerian rotation [Eq. (3) with $\delta=-1 / 3$ ] around spinless BH's.

With the increase of mass, while keeping the inner boundary close to the innermost stable circular orbit, the interval $\left(\kappa_{\min }, \kappa_{\max }\right)$ for which solutions were found, shifts downwards: both bounds for $\kappa$ go down. The choice of $\kappa=3$ always gives solutions.

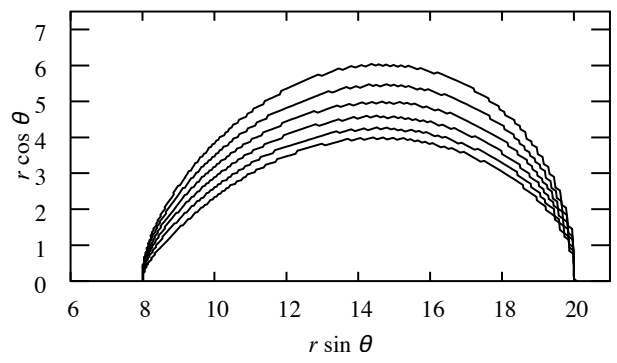

FIG. 1. Toroidal profiles for rotation around spin-less BH's, rotation law (3) with $\delta=-0.99,-0.8,-0.6,-0.4,-0.2,0$; toroids flatten with the decrease of $\delta$. Masses: $M_{\mathrm{BH}} \in$ $(1.076,1.079) ; M_{\mathrm{ADM}}=2.0$. Here $r_{1}=8$ and $r_{2}=20$.

Empirical experience, gained through numerical investigations, shows that the rotation law (11) is valid beyond the range of applicability that is suggested by the preceding derivation. One can consider fluid tori in the Keplerian motion around BH's with the spin parameter $a$, and the rotation curve (11):

$$
j(\Omega) \equiv-\frac{1}{2} \frac{d}{d \Omega} \ln \left(1-\left(b^{2} \Omega^{2}+3 w^{\frac{4}{3}} \Omega^{\frac{2}{3}}(1-b \Omega)^{\frac{4}{3}}\right)\right.
$$

with $b$ that might be different from $a$. All pairs $(a, b) \in$ $(-1,1) \times(-1,1)$ appear to be admissible (but see a discussion below). Setting $b=0$ in the pair $(a, b)(a \neq 0)$ converts (11) into (3); but this corresponds to the Keplerian motion of tori around spinning (if $a \neq 0$ ) or spinless black holes $(a=0)$. Thus the Keplerian version of the rotation law (3) is valid also for spinning BH's.

TABLE I. The first 2 columns give the innermost and outermost radii of tori, the third describes maximal values of the baryonic mass density, the fourth and fifth give $M_{\mathrm{T}}$ and $M_{\mathrm{BH}}$ respectively (with $\kappa=3$ ), and the final two columns show the minimal and maximal values of $\kappa$ for which solutions were found.

\begin{tabular}{ccccccc}
\hline \hline$r_{1}$ & $r_{2}$ & $\rho_{0}$ & $M_{\mathrm{T}}$ & $M_{\mathrm{BH}}$ & $\kappa_{\min }$ & $\kappa_{\max }$ \\
\hline 5 & 20 & $0.2 \times 10^{-4}$ & 0.005 & 1.000 & 3.0 & 4.9 \\
5 & 20 & $1.0 \times 10^{-4}$ & 0.078 & 1.008 & 2.9 & 4.8 \\
5 & 20 & $5.0 \times 10^{-4}$ & 1.1 & 1.120 & 1.5 & 4.0 \\
5 & 10 & $0.2 \times 10^{-3}$ & 0.007 & 1.001 & 3.0 & 5.2 \\
5 & 10 & $1.0 \times 10^{-3}$ & 0.11 & 1.017 & 2.7 & 5.0 \\
5 & 10 & $4.0 \times 10^{-3}$ & 1.0 & 1.176 & 0.8 & 3.9 \\
\hline \hline
\end{tabular}


V. NUMERICAL RESULTS: TORI AROUND SPINNING BH'S, ROTATION CURVE (3) WITH $\delta=-0.5$

We analyzed toroids circulating around BH's with the spin parameters $a=-0.5,0.0,0.5$. Resulting profiles are shown in Fig. 2 their shape depends rather weakly on spins. The enthalpy and mass density isolines are relatively insensitive to spins of BH's, similarly as their maximal values, which change by at most $5 \%$.

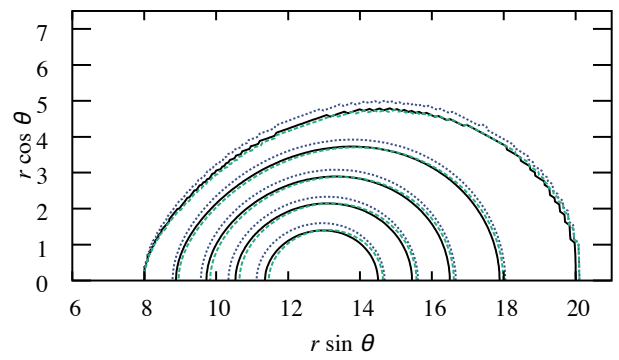

FIG. 2. Toroidal profiles for rotation around spinning BH's, rotation law (3) with $\delta=-0.5$ and spins $a=-0.5$ (broken green line, $M_{\mathrm{T}}=0.948$ ), $a=0.0$ (solid black line, $\left.M_{\mathrm{T}}=0.922\right), a=0.5$ (dotted blue line, $\left.M_{\mathrm{T}}=0.953\right)$. The asymptotic mass $M_{\mathrm{ADM}}=2.0$. The innermost and outermost coordinate radii are 8 and 20 , respectively. The internal isolines correspond to $h=1.02398,1.01799,1.01199,1.006$, with the outermost isoline representing $h=1+10^{-15}$.

TABLE II. Minimal masses of Keplerian tori for $\kappa=3$ and $b=0$. Here $r_{1}=8, r_{2}=20$ and $M_{\mathrm{BH}} \in(1.001,1003)$.

$\begin{array}{llllllllll}a & -0.9 & -0.8 & -0.7 & -0.6 & -0.5 & -0.4 & -0.3 & -0.2 & -0.1\end{array}$

\begin{tabular}{lllllllllll}
$M_{\mathrm{T}}$ & 0.143 & 0.127 & 0.111 & 0.086 & 0.075 & 0.057 & 0.045 & 0.034 & 0.014 \\
\hline
\end{tabular}

TABLE III. Minimal masses of Keplerian tori for $\kappa=3$ and $a=-0.5$. Here $r_{1}=5, r_{2}=20$.

\begin{tabular}{ccccc}
\hline \hline$b$ & -0.3 & -0.4 & -0.45 & -0.5 \\
$M_{\mathrm{T}}$ & $6.23 \times 10^{-2}$ & $3 \times 10^{-2}$ & $1.52 \times 10^{-2}$ & 0 \\
\hline \hline
\end{tabular}

\section{THE MASS GAP CONJECTURE}

Consider a $\mathrm{BH}$ with the spin parameter $a$ and a torus evolved according to 12 . Assume $\kappa=3$ and $\Omega>0$. Then there exists a mass gap in the spectrum of toroidal masses, if $b-a \geq 0$.

We show here two classes of numerical data that support this conjecture.

(i) The spin parameter $a$ is negative, $a \in[-0.1,-0.9]$, while the rotation parameter vanishes, $b=0$. Then it appears that tori may exist only if their mass is larger than a particular mass threshold. This mass gap goes to zero with vanishing $a$ (see Table II).

(ii) $a=-0.5$ and $b=-0.5,-0.45,-0.4,-0.3$. Toroids exist only if their mass is larger than a certain minimal value, which goes to zero with vanishing $b-a$ (Table III).

The existence of this mass gap is of interest from the mathematical point of view, since it means that the spacetime geometry produced by counter-rotating disks with the above specified rotation law does not tend to the Kerr geometry; solutions cease to exist when masses of toroids become too small. We have found that this mass threshold disappears for other values of $\kappa[16$.

\section{NUMERICS: TORI AROUND SPINNING BH'S, ROTATION CURVE (11)}

Figure 3 demonstrates that shapes of tori, their enthalpy and mass density, depend weakly on the value of the spin parameter $a$. The mass spectrum of tori starts from zero, as expected, since now $b-a=0$.

Both formulae (3) and (11) can be understood as a prescription for the spin-orbit interaction between BH's and fluid tori around them. The new rotation law of this letter can be more suitable than the former version [7] for the evolution of Keplerian disks around spinning BH's, since it gives the gapless mass spectrum of tori and does not contain any free parameters.

We have shown that the former law [7] can be numerically implemented within the puncture framework [15]. It is capable to describe compact $\mathrm{BH}$-torus systems that can be created in the merger of compact binaries consisting of pairs of neutron stars [3, 6, 21, 22, and that might exist also in some galactic centers [23, 24]. Our results agree with the earlier post-Newtonian analysis (see $[14,25,26]$ ); the relevant analysis will be published elsewhere. Self-gravitating fluid bodies can now be investigated in the regime of strong gravity for GR versions of the Keplerian rotation, including stationary disks in

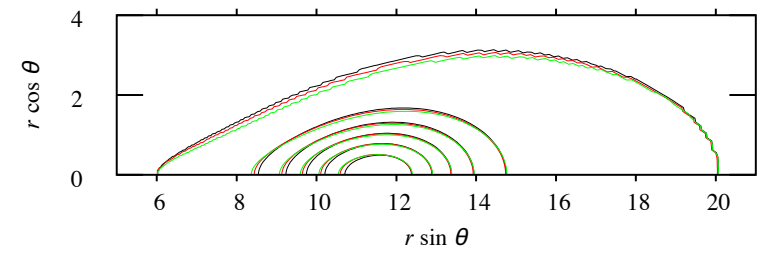

FIG. 3. Toroidal mass density profiles $\left(\rho=10^{-12}\right)$ and isolines $\rho=0.00002,0.00004,0.00006,0.00008,0.0001$ for the rotation law (11), $a=b=:-0.9$ (solid line); 0 (dotted line); 0.9 (dashed line). Masses: $M_{\mathrm{BH}} \in[1.001,1.009] ; M_{\mathrm{ADM}} \in$ $[1.101,1.110] ; M_{\mathrm{T}} \approx 0.10$. Here $r_{1}=6$ and $r_{2}=20$. 
tight accretion systems with central (spinless or spinning) BH's or rotating stars (with some adjustments of the rotation law in the vicinity of the symmetry axis). Our construction can be used in order to specify initial data for disk-BH configurations with viscosity; their evolution would model formation of ejecta in post-merger remains of two coalescing neutron stars.

\section{ACKNOWLEDGMENTS}

This research was carried out with the supercomputer "Deszno" purchased thanks to the financial support of the European Regional Development Fund in the framework of the Polish Innovation Economy Operational Program (contract no. POIG. 02.01.00-12-023/08). M.P. acknowledges partial support from the Grant No. K/DSC/004356. P.M. acknowledges the financial support of the Narodowe Centrum Nauki Grant No. DEC2012/06/A/ST2/00397.
[1] L. Baiotti and L. Rezzolla, Rep. Prog. in Phys. 80, 096901 (2017).

[2] B.P. Abbott et al., Phys. Rev. Lett. 119, 161101 (2017).

[3] W. Kastaun and F. Galeazzi, Phys. Rev. D 91, 064027 (2015).

[4] L. Rezzolla and P. Kumar, Astrophys. J. 802, 95 (2015).

[5] A. Endrizzi, R. Ciolfi, B. Giacomazzo, W. Kastaun, and T. Kawamura, Classical Quantum Gravity 33, 164001 (2016).

[6] M. Shibata, Sh. Fujibayashi, K. Hotokezaka, K. Kiuchi, K. Kyutoku, Y. Sekiguchi, and M. Tanaka, Phys. Rev. D96, 123012 (2017).

[7] P. Mach and E. Malec, Phys. Rev. D 91,124053 (2015).

[8] E. Butterworth and I. Ipser, Astrophys. J. 200,L103 (1975).

[9] J. M. Bardeen, Astrophys. J. 162, 71(1970); J. M. Bardeen and R. V. Wagoner, Astrophys. J. 167, 359 (1971).

[10] F. Galeazzi, S. Yoshida and Y. Eriguchi, Astron. Astrophys. 541, A156 (2012).

[11] K. Uryu, A. Tsokaros, F. Galeazzi, H. Hotta, M. Sugimura, K. Taniguchi, and S. Yoshida, Phys. Rev. D 93, 044056 (2016).

[12] K. Uryu, A. Tsokaros, L. Baiotti, F. Galeazzi, K. Taniguchi, and S. Yoshida, Phys. Rev. D 96, 103011 (2017).

[13] M. Ansorg and D. Petroff, Phys. Rev. D 72, 024019 (2005).

[14] J. Karkowski, P. Mach, E. Malec, M. Piróg, and N. Xie, Phys. Rev. D 94, 124041 (2016).

[15] M. Shibata, Phys. Rev. D 76, 064035 (2007).
[16] J. Karkowski, W. Kulczycki, P. Mach, E. Malec, A. Odrzywołek, and M. Piróg, Phys. Rev. D 97, 104017 (2018)

[17] W. Kastaun, F. Galeazzi, D. Alic, L. Rezzolla, and J. A. Font, Phys. Rev. D 88, 021501 (2013).

[18] L.G. Fishbone and V. Moncrief, Astrophys. J. 207, 962 (1976).

[19] We learned recently that a similar rotation law for test disks around Kerr BH's, in Boyer-Lindquist coordinates and with rigidly set $\kappa=1$, was proposed in J. -P. De Villiers, J. F. Hawley, and J. H. Krolik, Astrophys. J. 599, 1238 (2003).

[20] J. M. Bardeen, W. H. Press, and S. A. Teukolsky, Astrophys. J. 178, 347 (1972).

[21] F. Pannarale, A. Tonita, and L. Rezzolla, Astrophys. J. 727, 95 (2011)

[22] G. Lovelace, M. D. Duez, F. Foucart, L. E. Kidder, H. P. Pfeiffer, M. A. Scheel, and B. Szilagyi, Classical Quantum Gravity 30, 135004 (2013).

[23] J. Bardeen and J. Petterson, Astrophys. J. 195, L65 (1975).

[24] J. Moran, Proceedings of the conference held 18-21 June, 2007, Frontiers of Astrophysics: A Celebration of NRAO's 50th Anniversary ASP Conference Series, edited by A. H. Bridle, J. J. Condon, and G. C. Hunt, vol. 395 (2008), p. 87.

[25] P. Jaranowski, P. Mach, E. Malec, and M. Piróg, Phys. Rev. D 91, 024039 (2015).

[26] J. Knopik, P. Mach, and E. Malec, Acta Phys. Pol. B 46, 2451 (2015). 